\title{
Perfil Clínico e Desfechos em 30 Dias de Pacientes Portadores de Valva Aórtica Bicúspide Submetidos à Cirurgia em Valva Aórtica e/ou Aorta
}

\author{
Clinical Profile and 30-Day Outcomes of Patients with Bicuspid Aortic Valve Undergoing Aortic Valve and/or \\ Aorta Surgery
}

Marcelo Kirschbaum, ${ }^{10}$ Vitor Emer Egypto Rosa, ${ }^{10}$ Brunna Pileggi Azevedo Sampaio, ${ }^{10}$ Gabriela Thevenard, ${ }^{1}$ Nádia Romanelli Quintanilha, ${ }^{1}$ João Ricardo Cordeiro Fernandes, ${ }^{1}$ Antonio de Santis, ${ }^{1}$ Tarso Duenhas Accorsi, ${ }^{1}$ Roney Orismar Sampaio, ${ }^{1}$ Flavio Tarasoutchi ${ }^{10}$

Unidade Clínica de Valvopatias - Instituto do Coração (InCor) do Hospital das Clínicas da Faculdade de Medicina da Universidade de São Paulo (HC-FMUSP), ${ }^{1}$ São Paulo, SP - Brasil

\section{Resumo}

Fundamento: A válvula aórtica bicúspide (VAB) atinge de 0,5 a $2 \%$ da população e está associada a alterações valvares e de aorta. Há carência de estudos sobre o perfil desses pacientes na população brasileira.

Objetivo: Descrever o perfil de pacientes com VAB submetidos à cirurgia valvar e/ou de aorta em um centro cardiológico terciário, assim como os desfechos relacionados à intervenção.

Métodos: Coorte retrospectiva incluindo 195 pacientes (idade média $54 \pm 14$ anos, 73,8\% do sexo masculino) com diagnóstico de VAB submetidos à abordagem cirúrgica (valvar e/ou de aorta) no período de 2014 a 2019. Foram avaliados dados clínicos, ecocardiográficos e tomográficos, além das características da intervenção e eventos em 30 dias. $\mathrm{O}$ valor de $\mathbf{p}<0,05$ foi considerado estatisticamente significante.

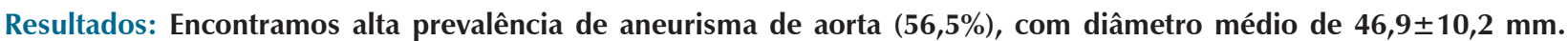
Insuficiência aórtica importante foi encontrada em 25,1\% e estenose aórtica importante em 54,9\%. Cirurgia isolada em valva aórtica foi realizada em 48,2\%, cirurgia isolada de aorta em 6,7\% e cirurgia combinada em 45,1\%. A mortalidade em 30 dias foi de $8,2 \%$. Na análise multivariada, os fatores preditores de desfecho combinado em 30 dias (morte, fibrilação atrial e reoperação) foram idade (OR 1,044, IC 95\% 1,009-1,081, p=0,014) e o índice de massa do ventrículo esquerdo (OR 1,009, IC 95\% 1,000-1,018, $p=0,044)$.

Conclusão: Pacientes com VAB abordados no nosso serviço apresentam uma maior incidência de aortopatia, com a necessidade adicional de avaliação da aorta com tomografia computadorizada ou ressonância magnética.

Palavras-chave: Valva Aórtica; Cirurgia Torácica; Estenose da Valva Aórtica.

\footnotetext{
Abstract

Background: The bicuspid aortic valve (BAV) affects 0.5 to $2 \%$ of the population and is associated with valve and aortic alterations. There is a lack of studies on the profile of these patients in the Brazilian population.
}

Objective: To describe the profile of patients with BAV undergoing valve and/or aortic surgery in a tertiary cardiology center, in addition to the outcomes related to the intervention.

Methods: Retrospective cohort including 195 patients (mean age $54 \pm 14$ years, $73.8 \%$ male) diagnosed with BAV who underwent surgical approach (valvular and/or aorta) from 2014 to 2019. Clinical data, echocardiographic and tomographic studies were evaluated, as well as characteristics of the intervention and events in 30 days. A value of $p<0.05$ was considered statistically significant.

Results: We found a high prevalence of aortic aneurysm (56.5\%), with a mean diameter of $46.9 \pm 10.2 \mathrm{~mm}$. Major aortic regurgitation was found in $25.1 \%$ and major aortic stenosis in $54.9 \%$. Isolated aortic valve surgery was performed in $48.2 \%$, isolated aortic surgery in $6.7 \%$ and combined surgery in $45.1 \%$. The 30-day mortality was $8.2 \%$. In the multivariate analysis, the predictors of the combined outcome at 30 days (death, atrial fibrillation and reoperation) were age (OR 1.044, 95\% Cl 1.009-1.081, $p=0.014)$ and left ventricular mass index $(O R$ 1.009, 95\% Cl 1.000-1.018, $p=0.044$ ). 
Conclusion: Patients with BAV approached in our service have a higher incidence of aortopathy, with the additional need to evaluate the aorta with computed tomography or magnetic resonance imaging.

Keywords: Aortic Valve; Thoracic Surgery; Aortic Valve Stenosis.

Full texts in English - http://www.arquivosonline.com.br

\section{Introdução}

A válvula aórtica bicúspide (VAB) é a doença cardíaca congênita mais prevalente, atingindo de 0,5 a $2 \%$ da população mundial. ${ }^{1-3}$ A expectativa de vida assemelha-se a da população geral, porém esses pacientes apresentam alterações hemodinâmicas, celulares, moleculares e genéticas que estão intrinsecamente relacionadas a repercussões na válvula aórtica e na aorta, com necessidade de intervenção cirúrgica precoce. ${ }^{4-7}$ A prevalência e progressão destas alterações são proporcionais à idade, sendo o maior risco de eventos cardiovasculares em pacientes com idade maior que 30 anos. ${ }^{8}$

Tal complexidade etiopatogênica da VAB gera uma heterogeneidade de apresentações clínicas. Além disso, há uma carência de informações sobre o perfil clínico dos pacientes portadores de VAB submetidos à cirurgia cardíaca, principalmente da população brasileira.

\section{Objetivo}

O objetivo desse trabalho é descrever o perfil de pacientes com VAB submetidos à cirurgia valvar e/ou de aorta em um centro cardiológico terciário, além dos desfechos relacionados à intervenção.

\section{Métodos}

População estudada: Coorte retrospectiva de pacientes acima de 18 anos com diagnóstico de VAB submetidos à abordagem cirúrgica da aorta e/ou válvula aórtica, entre os anos de 2014 a 2019. Todos os pacientes foram submetidos à ecocardiografia transtorácica e avaliação da aorta ascendente e arco aórtico por tomografia computadorizada ou ressonância magnética antes da cirurgia. A indicação cirúrgica foi baseada nos protocolos institucionais, de acordo com as diretrizes vigentes para tratamento de valvopatias e das doenças de aorta. ${ }^{9,10}$ Foram excluídos pacientes sem documentação da avaliação de aorta ou ecocardiograma pré-procedimento. $\mathrm{O}$ protocolo do estudo foi revisado e aprovado pelo comitê de ética institucional local.

Protocolo do estudo: Foram avaliados dados préoperatórios da população como idade, sexo, medicações em uso, presença de sintomas, risco cirúrgico pelo EuroSCORE II, comorbidades, características anatômicas da aorta por tomografia computadorizada ou ressonância magnética, anatomia cardíaca e valvar pelo ecocardiograma e dados laboratoriais de hemoglobina e creatinina. Nos desfechos de 30 dias, foram analisados dados de mortalidade e complicações perioperatórias, além de desfecho combinado em 30 dias de mortalidade, fibrilação atrial e reabordagem cirúrgica.

Análise estatística: Foi utilizado para análise estatística o programa SPSS versão 26 (IBM, Armonk, NY), sendo realizadas análises descritivas simples de frequência e porcentagem para variáveis categóricas, com descrição de média e desvio padrão ou mediana e intervalo interquartil para variáveis contínuas. Foi analisada a distribuição de normalidade dos dados por meio do teste de Kolmogorov-Smirnov. Para análise comparativa entre os grupos, foi utilizado teste de qui-quadrado ou teste exato de Fisher para avaliação das variáveis categóricas, conforme apropriado. Para comparação de variáveis contínuas, foi utilizado teste $\mathrm{T}$ de Student não pareado ou teste de Mann-Whitney, conforme apropriado. A análise univariada de preditores relacionados ao desfecho combinado em 30 dias de mortalidade, fibrilação atrial e reabordagem, foi realizada com regressão logística binária. $\mathrm{Na}$ análise univariada, aquelas com valor de $\mathrm{p}<0,05$ foram selecionadas e inseridas no modelo multivariado de regressão logística binária. A relação da presença de estenose ou insuficiência aórtica com o índice de massa do ventrículo esquerdo foi avaliada através do método de regressão linear, sendo verificados os pressupostos necessários para o uso dessa técnica (variabilidade e distribuição dos erros). O valor de $p<0,05$ foi considerado estatisticamente significante.

\section{Resultados}

Características da população: Foram incluídos 195 pacientes consecutivos portadores de VAB submetidos à cirurgia neste período. A idade média foi de $54 \pm 14$ anos, maioria do sexo masculino e com alta prevalência de comorbidades como hipertensão arterial sistêmica, diabetes e doença renal crônica. As características da população estudada encontram-se na Tabela 1. Na avaliação da aorta, 187 (95,9\%) pacientes foram submetidos à realização de tomografia computadorizada e o restante $(4,1 \%)$ realizaram ressonância magnética, sendo que $76,4 \%$ apresentavam ectasia aórtica (aorta $>38 \mathrm{~mm}$ ) e $56,5 \%$ apresentavam aneurisma de aorta (aorta $>45 \mathrm{~mm}$ ), sendo o diâmetro médio da aorta ascendente de 46,9 910,2 mm (Figura 1). Pela avaliação ecocardiográfica, a média da fração de ejeção do ventrículo esquerdo pré-operatória foi de $59 \pm 11 \%$, com insuficiência aórtica importante em $25,1 \%$ e estenose aórtica importante em 54,9\%. Pacientes com estenose aórtica apresentaram gradiente transaórtico médio de 49,1 \pm 17,0 mmHg e área valvar aórtica de 0,79 $\pm 0,19 \mathrm{~cm}^{2}$. A indicação cirúrgica pela valvopatia aórtica importante ocorreu em 62,6\% dos casos, 33,3\% indicado por aortopatia importante e o restante por coronariopatia ou valvopatia mitral.

A dissecção aguda de aorta foi descrita em 5,6\% dos pacientes, sendo que estes apresentavam maiores diâmetros de aorta do que os que não apresentaram dissecção aguda $(54,95 \pm 21,36$ vs 46,81 $\pm 8,81 \mathrm{~mm}, \mathrm{p}=0,010)$.

Características cirúrgicas e desfechos clínicos: Os dados relacionados à cirurgia e eventos clínicos estão descritos na Tabela 2. Em 45,1\% dos casos, o procedimento cirúrgico foi 


\begin{tabular}{|c|c|}
\hline Variáveis & $\mathrm{n}=195$ \\
\hline \multicolumn{2}{|l|}{ Características clínicas } \\
\hline Idade, anos & $54,7 \pm 14,1$ \\
\hline Sexo feminino & $51(26,2)$ \\
\hline Superfície corpórea, $\mathrm{m}^{2}$ & $1,88 \pm 0,21$ \\
\hline Hipertensão arterial sistêmica & $111(56,9)$ \\
\hline Diabetes & $25(12,8)$ \\
\hline Fibrilação atrial prévia & $15(7,7)$ \\
\hline Doença renal crônica* & $44(22,6)$ \\
\hline Doença arterial coronária & $39(46,7)$ \\
\hline Endocardite prévia & $9(4,6)$ \\
\hline Angina & $46(23,6)$ \\
\hline Dispneia NYHA III ou IV & $112(59,1)$ \\
\hline EuroSCORE II, \% & $1,61(0,93-3,02)$ \\
\hline Betabloqueador & $90(46,2)$ \\
\hline Diuréticos & $95(48,7)$ \\
\hline IECA & $59(30,3)$ \\
\hline BRA & $64(32,8)$ \\
\hline Estatinas & $75(38,5)$ \\
\hline \multicolumn{2}{|l|}{ Laboratório } \\
\hline Hemoglobina, mg/dL & $13,9 \pm 1,7$ \\
\hline Creatinina, $\mathrm{mg} / \mathrm{dL}$ & $1,14 \pm 0,56$ \\
\hline \multicolumn{2}{|l|}{ Características da aorta } \\
\hline Maior diâmetro de aorta torácica, $\mathrm{mm}$ & $46,9 \pm 10,2$ \\
\hline Maior diâmetro de aorta torácica indexado, $\mathrm{mm} / \mathrm{m}^{2}$ & $25 \pm 6$ \\
\hline Diâmetro de aorta $>38 \mathrm{~mm}$ & $149(76,4)$ \\
\hline Diâmetro de aorta > 45 mm & $100(56,5)$ \\
\hline Dissecção aguda & $11(5,6)$ \\
\hline Coarctação & $11(5,6)$ \\
\hline \multicolumn{2}{|l|}{ Ecocardiograma } \\
\hline Seio Aórtico, mm & $37,4 \pm 6,8$ \\
\hline Diâmetro do átrio esquerdo, $\mathrm{mm}$ & $40,5 \pm 7,2$ \\
\hline Septo, mm & $12 \pm 4$ \\
\hline Parede posterior, mm & $11 \pm 1$ \\
\hline Índice de massa de VE, $\mathrm{g} / \mathrm{m}^{2}$ & $142 \pm 53$ \\
\hline Diâmetro diastólico de VE, mm & $56,8 \pm 10,7$ \\
\hline Diâmetro sistólico de VE, mm & $38,3 \pm 9,4$ \\
\hline Fração de ejeção de VE, \% & $59 \pm 11$ \\
\hline Área valvar aórtica, $\mathrm{cm}^{2} \dagger$ & $0,82 \pm 0,22$ \\
\hline Gradiente transaórtico máximo, mmHg† & $54 \pm 33$ \\
\hline Gradiente transaórtico médio, mmHg $†$ & $42 \pm 19$ \\
\hline Insuficiência aórtica importante & $48(25,1)$ \\
\hline Estenose aórtica importante & $104(53,3)$ \\
\hline Dupla lesão aórtica importante & $16(15,5)$ \\
\hline \multicolumn{2}{|l|}{ Indicação cirúrgica } \\
\hline Estenose aórtica importante & $78(40,0)$ \\
\hline Insuficiência aórtica importante & $44(22,6)$ \\
\hline Aorta & $65(33,3)$ \\
\hline Coronária ou valva mitral & $8(4,1)$ \\
\hline
\end{tabular}

Dados apresentados em média \pm desvio padrão, mediana (intervalo interquartil) ou n (\%). *Doença renal crônica foi definida por clearance de creatinina $<60 \mathrm{ml} / \mathrm{kg} / \mathrm{min}$. †Parâmetros descritos apenas em pacientes portadores de estenose aórtica. BRA: bloqueador de receptor de angiotensina II; IECA: Inibidor da Enzima Conversora de Angiotensina; NYHA: New York Heart Association; VE: ventrículo esquerdo. 

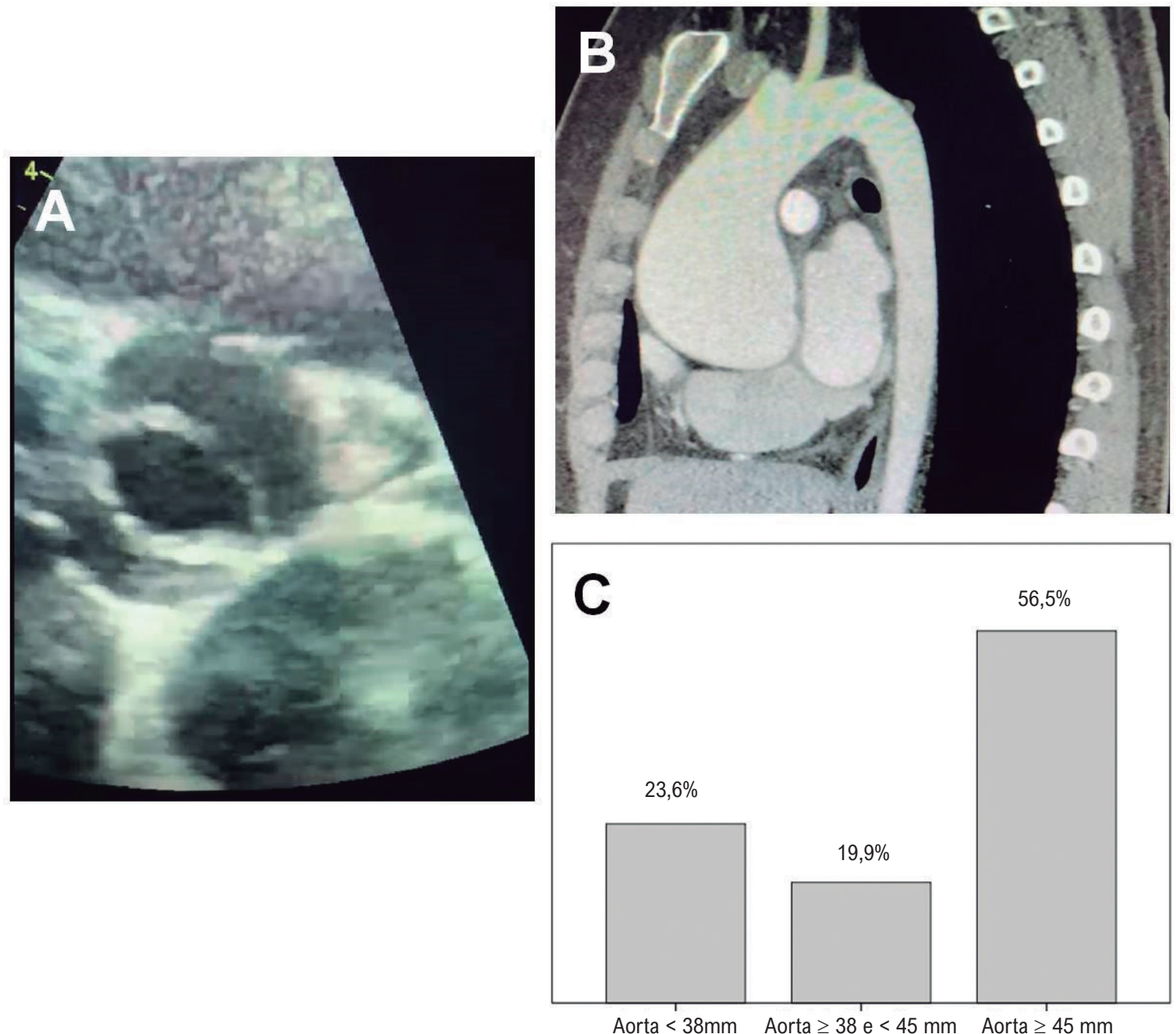

Figura 1 - A) Janela paraesternal transversal de ecocardiograma transtorácico evidenciando válvula aórtica bicúspide. B) Angiotomografia computadorizada de aorta evidenciando dilatação de aorta ascendente. C) Incidência de pacientes com válvula aórtica bicúspide e aorta menor que $38 \mathrm{~mm}$, aorta entre 28 e $45 \mathrm{~mm}$, e aorta maior ou igual a $45 \mathrm{~mm}$.

combinado de aorta e valva aórtica e, desses, 53,4\% foi submetido à cirurgia de Bentall de Bono, 33\% à cirurgia de Bentall de Bono modificada com implante de prótese biológica e os demais submetidos à cirurgia com preservação de valva aórtica. Em 94 (48,2\%) pacientes foi realizada cirurgia isolada em valva aórtica e em $13(6,7 \%)$ cirurgia isolada de aorta. Uma prótese biológica foi implantada em $60,4 \%$ dos pacientes nos quais a válvula aórtica foi abordada, prótese mecânica em 30,2\%, plástica de válvula aórtica em 8,8\% e 1 paciente foi submetido à abordagem transcateter (TAVI). A mortalidade em 30 dias na população foi de $8,2 \%$, maior do que a prevista pelo EuroSCORE II $(1,61[0,93-3,02] \%)$. No período pós-operatório, 21,5\% dos pacientes apresentaram insuficiência renal aguda, 15,7\% fibrilação atrial e 9,7\% deles necessitaram de reoperação. Os eventos de acordo com o tipo de lesão valvar (estenose aórtica importante, insuficiência aórtica importante, dupla lesão importante e dupla lesão moderada) estão descritos na Tabela Suplementar 1.

Preditores do desfecho combinado: Os preditores do desfecho combinado em 30 dias de morte, fibrilação atrial e reoperação na análise univariada estão descritos na Tabela 3 e Tabela Suplementar 2. Na análise multivariada, idade e índice de massa do ventrículo esquerdo mantiveram-se como preditores independentes do desfecho combinado. Embora a presença de estenose ou insuficiência aórtica não seja preditora de eventos, encontramos relação dessas variáveis com o índice de massa do ventrículo esquerdo $(B=18,52$, IC 95\% $=3,96-33,09, p=0,013$ e $B=61,80$, IC 95\% $=44,73$ $78,87, p<0,001$; respectivamente). A análise multivariada excluindo o paciente submetido ao TAVI encontrou os mesmos preditores de desfecho combinado descritos anteriormente e está demonstrada na Tabela Suplementar 3. 


\begin{tabular}{|c|c|}
\hline Variável & $n=195$ \\
\hline \multicolumn{2}{|l|}{ Procedimento } \\
\hline Cirurgia combinada (Aorta e Valva aórtica) & $88(45,1)$ \\
\hline Bentall de Bono & $47(53,4)$ \\
\hline Bentall de Bono modificado & $29(33,0)$ \\
\hline Plástica valvar aórtica & $12(13,6)$ \\
\hline Cirurgia em aorta isolada & $13(6,7)$ \\
\hline Cirurgia em valva aórtica & $94(48,2)$ \\
\hline Prótese biológica & $80(85,1)$ \\
\hline Prótese mecânica & $9(9,5)$ \\
\hline Plástica valvar & $4(4,2)$ \\
\hline TAVI & $1(1,0)$ \\
\hline Revascularização miocárdica associada & $24(12,3)$ \\
\hline \multicolumn{2}{|l|}{ Desfechos em 30 dias } \\
\hline Mortalidade & $16(8,2)$ \\
\hline Sangramento & $28(14,4)$ \\
\hline Hemotransfusão & $41(21)$ \\
\hline Insuficiência renal aguda* & $42(21,5)$ \\
\hline Reoperação & $19(9,7)$ \\
\hline Acidente vascular cerebral & $4(2,1)$ \\
\hline Tamponamento cardíaco & $8(4,1)$ \\
\hline Desfecho combinado (morte + fibrilação atrial + reoperação) & $55(28,2)$ \\
\hline Tempo de internação em UTI, dias & $5,1 \pm 5,8$ \\
\hline
\end{tabular}

Dados apresentados em média \pm desvio padrão ou n (\%). *Insuficiência renal aguda definida como aumento de creatinina $\geq 0,3$ mg/dl. TAVI: Implante de bioprótese aórtica transcateter, do inglês Transacatheter aortic valve replacement; UTI: Unidade de Terapia Intensiva.

Tabela 3 - Análise de preditores para o desfecho composto em 30 dias de morte, fibrilação atrial e reabordagem

\begin{tabular}{lcccc}
\hline \multirow{2}{*}{ Variável } & \multicolumn{2}{c}{ Análise univariada } & \multicolumn{2}{c}{ Análise multivariada } \\
\cline { 2 - 5 } & OR (IC 95\%) & $\mathbf{p}$ & OR (IC 95\%) & $\mathbf{p}$ \\
\hline Idade, anos & $1,051(1,023-1,078)$ & $<0,001$ & $1,044(1,008-1,082)$ & 0,016 \\
\hline Superfície corpórea, m & $0,214(0,047-0,974)$ & 0,046 & $0,178(0,019-1,658)$ & 0,130 \\
\hline Hemoglobina, mg/dL & $0,812(0,673-0,978)$ & 0,029 & $0,871(0,680-1,116)$ & 0,276 \\
\hline Bloqueador de receptor de angiotensina II & $1,916(1,003-3,660)$ & 0,049 & $0,680(0,297-1,557)$ & 0,362 \\
\hline Diâmetro de átrio esquerdo, mm & $1,078(1,028-1,131)$ & 0,002 & $1,072(0,995-1,155)$ & 0,067 \\
\hline Índice de massa, g/m² & $1,007(1,001-1,014)$ & 0,017 & $1,009(1,000-1,018)$ & 0,044 \\
\hline Fração de ejeção do VE, \% & $0,960(0,933-0,987)$ & 0,004 & $0,981(0,945-1,018)$ & 0,305 \\
\hline Insuficiência tricúspide moderada ou importante & $6,550(1,923-22,309)$ & 0,003 & $0,528(0,095-2,950)$ & 0,467 \\
\hline Insuficiência mitral moderada ou importante & $2,603(1,035-6,549)$ & 0,042 & $2,646(0,633-11,069)$ & 0,183 \\
\hline Cirurgia em valva aórtica & $3,257(1,042-10,175)$ & 0,042 & $2,972(0,505-17,504)$ & 0,229 \\
\hline
\end{tabular}

OR: odds ratio; VE: ventriculo esquerdo. 
Comparação de acordo com a indicação de intervenção: A comparação dos pacientes de acordo com indicação de cirurgia pelo diâmetro da aorta ou indicação pela valvopatia encontra-se na Tabela 4. Pacientes em que a intervenção foi indicada pela doença da aorta eram menos sintomáticos e com menor remodelamento cardíaco, com índice de massa de VE menor, menor diâmetro de átrio esquerdo, septo e parede posterior menos espessos. Conforme esperado, os pacientes com indicação pela doença da aorta apresentaram maiores diâmetros de aorta e diâmetros indexados de aorta. Os pacientes indicados pela valvopatia apresentaram maior proporção de cirurgia combinada. Não encontramos diferenças entre os grupos em relação a desfechos.

\section{Discussão}

Os principais achados desse estudo foram: (1) $76,4 \%$ dos pacientes com VAB apresentavam aortopatia associada, (2) por ser um centro terciário, ressalta-se uma elevada morbidade, com $56,9 \%$ de hipertensos e $46,7 \%$ de pacientes com doença arterial coronariana, deste modo encontramos uma mortalidade na intervenção maior do que a prevista pelo EuroSCORE II e (3) a idade e índice de massa foram preditores do desfecho combinado de morte, fibrilação atrial e reoperação em 30 dias.

A VAB é uma alteração na embriogênese da valva aórtica não totalmente esclarecida, porém com diversas teorias acerca de sua origem, desde alteração no fluxo transvalvar fetal levando a falha na separação das cúspides, até teorias mais atuais relacionando fatores genéticos e falha de migração celular em algumas fases da embriogênese. ${ }^{11-13} \mathrm{~A}$ fusão das cúspides leva ao turbilhonamento do fluxo valvar, predispondo, assim, à degeneração precoce da valva aórtica. O fluxo turbilhonado também é responsável por um estresse assimétrico na parede da aorta, podendo predispor à dilatação da mesma. ${ }^{4}$ Além dessa alteração hemodinâmica no fluxo da via de saída, explicando a aortopatia associada à degeneração valvar, também ocorrem alterações microscópicas como a redução da fibrilina-1, disrupção da matrix, apoptose e aumento de metaloproteinases, justificando a presença de dilatação aórtica nos pacientes com função valvar inalterada. ${ }^{6,7,14}$

A indicação de abordagem na VAB pode estar relacionada à valvopatia aórtica anatomicamente importante associada a sintomas ou complicadores - indicação essa semelhante à de outras valvopatias ou aortopatia propriamente dita. A indicação de abordagem de aorta varia conforme o caso. Nos pacientes com dilatação aórtica sem valvopatia, é indicado acompanhamento frequente daqueles com diâmetro de aorta maior que $45 \mathrm{~mm}$ ou aumento de 0,3cm/ano. As diretrizes da European Society of Cardiology para diagnóstico e manejo de doenças de aorta de 2014 indicam abordagem para pacientes com diâmetro de aorta $>55 \mathrm{~mm}$ isoladamente e $>50 \mathrm{~mm}$ na presença de fatores de risco. ${ }^{10}$ As diretrizes da American Heart Association não definem valor de corte específico para abordagem isolada de aorta, orientando avaliação caso a caso de pacientes com diâmetro de aorta entre 40 e $50 \mathrm{~mm} .{ }^{15}$ Ambas diretrizes orientam abordagem da aorta com diâmetro $>45 \mathrm{~mm}$ se indicada intervenção primária valvar aórtica. ${ }^{10,15}$
Em nosso estudo, 93\% dos pacientes apresentarem valvopatia com indicação de intervenção, porcentagem semelhante ao estudo de Tzemos et al $(95,7 \%) .{ }^{8}$ Já em relação à incidência de aneurisma de aorta, existe grande variabilidade na literatura que pode ser explicada, dentre outros fatores, pela extrema heterogeneidade na definição de dilatação de aorta, variando entre 40 e $45 \mathrm{~mm} .{ }^{16-18}$ Apesar disso, a prevalência de aneurisma de aorta definido por aorta maior que $45 \mathrm{~mm}$, na nossa população, superou o descrito pela literatura (56,5\% vs. $20-30 \%$, respectivamente), reforçando a necessidade de avaliação da aorta com tomografia computadorizada ou ressonância magnética em todos pacientes portadores de VAB. , $19,20^{2}$ Nossa população apresentou alta prevalência de hipertensão arterial sistêmica, diabetes mellitus e doença arterial coronariana se comparado a outros estudos com pacientes com VAB. ${ }^{5,8} \mathrm{Um}$ achado relevante foi a alta incidência de dissecção aguda (5,6\%), descrita na literatura entre $0,5-1 \%$ dos pacientes com VAB em diversos estudos de desfecho cirúrgico e de seguimento a longo prazo. ${ }^{8,14} \mathrm{Em}$ consonância com a literatura, identificamos que tais pacientes com dissecção apresentavam maiores diâmetros de aorta do que aqueles sem tal alteração (54,9 $\pm 21,3$ vs. 46,8 \pm 8,8 $\mathrm{mm}, \mathrm{p}=0,010){ }^{20}$

Ressalta-se que a cirurgia combinada (aorta + válvula) também não esteve associada a pior prognóstico quando comparada à cirurgia valvar isolada. Ademais, os pacientes da nossa casuística apresentaram mortalidade em 30 dias maior que a predita pelo EuroSCORE II (8,2\% vs. 2,77 $24,07 \%$, respectivamente). Além do fato do EuroSCORE II não ter validação específica para a população brasileira com VAB, a elevada mortalidade ainda pode ser justificada por um viés de seleção, dado que nosso centro é de referência nacional e há uma tendência de atendimento de pacientes mais sintomáticos $(24,1 \%$ em classe funcional III/IV), com maior incidência de comorbidades (46,7\% com doença arterial coronária) e com maior repercussão cardíaca (média do índice de massa de ventrículo esquerdo de $142 \pm 53 \mathrm{~g} / \mathrm{m}^{2}$ ).

O aumento do índice de massa do ventrículo esquerdo e a idade foram identificados como preditores independentes de eventos pós-operatórios, sendo esta última também descrita em outras coortes observacionais de pacientes com VAB. ${ }^{8,19,21}$ Tais estudos também demonstram impacto da degeneração valvar no prognóstico, o que não foi confirmado em nosso estudo na análise multivariada. Entretanto, o aumento do índice de massa do ventrículo esquerdo teve correlação com a presença de estenose e insuficiência aórtica importantes, sendo um marcador indireto da repercussão valvar nas câmaras cardíacas esquerdas.

\section{Limitações}

A principal limitação desse estudo é inerente ao seu desenho de caráter observacional. Dessa forma, dados que poderiam influenciar negativamente o resultado cirúrgico e impactar em eventos (como tempo de circulação extracorpórea, tempo de internação hospitalar, uso de drogas vasoativas, uso de suporte circulatório, taxa de infecção, dentre outros) não estiveram disponíveis para a análise em todos os pacientes. Além disso, o seguimento de curto prazo não nos permite extrapolar nossos achados para além 


\begin{tabular}{|c|c|c|c|}
\hline Variáveis & $\begin{array}{l}\text { Indicação pelo diâmetro } \\
\text { da aorta }(n=65)\end{array}$ & $\begin{array}{l}\text { Indicação pela valvopatia } \\
\qquad(n=130)\end{array}$ & $\mathbf{p}$ \\
\hline \multicolumn{4}{|l|}{ Características clínicas } \\
\hline Idade, anos & $57,3 \pm 14,5$ & $53,4 \pm 13,8$ & 0,072 \\
\hline Superfície Corpórea, $\mathrm{m}^{2}$ & $1,88 \pm 0,22$ & $1,88 \pm 0,21$ & 0,917 \\
\hline Sexo feminino & $14(21,5)$ & $37(28,5)$ & 0,300 \\
\hline Hipertensão arterial sistêmica & $43(66,2)$ & $68(52,3)$ & 0,066 \\
\hline Diabetes mellitus & $8(12,2)$ & $17(13,1)$ & 0,880 \\
\hline Dislipidemia & $21(32,3)$ & $37(28,5)$ & 0,580 \\
\hline Doença renal crônica* & $20(30,8)$ & $24(18,5)$ & 0,053 \\
\hline EuroSCORE II, \% & $1,96(0,97-4,43)$ & $1,35(0,89-2,66)$ & 0,045 \\
\hline \multicolumn{4}{|l|}{ Laboratório } \\
\hline Hemoglobina, mg/dl & $14,0 \pm 1,6$ & $13,8 \pm 1,7$ & 0,395 \\
\hline Creatinina, mg/dl & $1,23 \pm 0,82$ & $1,10 \pm 0,36$ & 0,132 \\
\hline \multicolumn{4}{|l|}{ Sintomas } \\
\hline Angina & $13(20)$ & $33(25,4)$ & 0,520 \\
\hline Dispneia NYHA III e IV & $23(35,4)$ & $89(68,4)$ & $<0,001$ \\
\hline \multicolumn{4}{|l|}{ Medicações } \\
\hline Betabloqueador & $42(64,6)$ & $48(36,9)$ & $<0,001$ \\
\hline IECA & $16(24,6)$ & $43(33,1)$ & 0,250 \\
\hline BRA & $24(36,9)$ & $40(30,8)$ & 0,349 \\
\hline Espironolactona & $2(3,1)$ & $18(13,8)$ & 0,020 \\
\hline Diurético de alça & $27(41,5)$ & $68(52,3)$ & 0,185 \\
\hline \multicolumn{4}{|l|}{ Característica da Aorta } \\
\hline Maior diâmetro da aorta & $53,6 \pm 11,1$ & $43,1 \pm 7,4$ & $<0,001$ \\
\hline Maior diâmetro da aorta indexado & $28,7 \pm 6,9$ & $23,0 \pm 4,8$ & $<0,001$ \\
\hline \multicolumn{4}{|l|}{ Ecocardiograma } \\
\hline Seio Aórtico, mm & $41,0 \pm 7,1$ & $35,7 \pm 6,0$ & $<0,001$ \\
\hline Diâmetro do átrio esquerdo, $\mathrm{mm}$ & $38,9 \pm 6,7$ & $41,2 \pm 7,3$ & 0,035 \\
\hline Septo, mm & $11,0 \pm 1,7$ & $12,3 \pm 4,8$ & 0,012 \\
\hline Parede posterior do VE, $\mathrm{mm}$ & $10,1 \pm 1,5$ & $11,0 \pm 1,9$ & 0,001 \\
\hline Índice de massa de VE, $\mathrm{g} / \mathrm{m}^{2}$ & $126,1 \pm 44,7$ & $150,8 \pm 55,3$ & 0,002 \\
\hline Diâmetro diastólico do VE, mm & $54 \pm 9$ & $57 \pm 11$ & 0,064 \\
\hline Diâmetro sistólico do VE, mm & $36 \pm 8$ & $39 \pm 9$ & 0,136 \\
\hline Fração de ejeção do VE, \% & $60 \pm 8$ & $58 \pm 12$ & 0,089 \\
\hline Gradiente transaórtico médio, $\mathrm{mmHg}$ & $34 \pm 18$ & $44 \pm 18$ & 0,019 \\
\hline Área valvar aórtica, $\mathrm{cm}^{2}$ & $0,91 \pm 0,27$ & $0,80 \pm 0,20$ & 0,137 \\
\hline Estenose aórtica importante & $18(27,7)$ & $86(66,2)$ & $<0,001$ \\
\hline Insuficiência Aórtica importante & $10(15,4)$ & $38(29,2)$ & 0,047 \\
\hline Insuficiência Tricúspide moderada ou importante & $3(4,6)$ & $10(7,7)$ & 0,554 \\
\hline Insuficiência Mitral moderada ou importante & $3(4,6)$ & $18(13,8)$ & 0,059 \\
\hline \multicolumn{4}{|l|}{ Cirurgia } \\
\hline Aorta isolada & $13(20)$ & $0(0)$ & $<0,001$ \\
\hline Valva aórtica isolada & $0(0)$ & $95(73,1)$ & $<0,001$ \\
\hline Cirurgia combinada & $52(80)$ & $35(26,9)$ & $<0,001$ \\
\hline \multicolumn{4}{|l|}{ Desfecho em 30 dias } \\
\hline Morte & $5(7,7)$ & $11(8,5)$ & 0,854 \\
\hline Fibrilação atrial pós-operatório & $8(13,6)$ & $23(17,6)$ & 0,388 \\
\hline Reabordagem & $8(12,3)$ & $11(8,5)$ & 0,403 \\
\hline Desfecho combinado (morte + fibrilação atrial + reoperação) & $17(26,2)$ & $38(29,2)$ & 0,653 \\
\hline
\end{tabular}

Dados apresentados em média \pm desvio padrão, mediana (intervalo interquartil) ou $n$ (\%). *Doença renal crônica foi definida por clearance de creatinina <60 ml/kg/min. BRA: bloqueador de receptor de angiotensina II; IECA: Inibidor da Enzima Conversora de Angiotensina; NYHA: New York Heart Association; VE: ventrículo esquerdo. 
do período de 30 dias. Entretanto, o número de pacientes avaliados é grande para a patologia em questão, sendo a maior casuística da literatura nacional até o momento. Outro viés decorre do fato de nossa instituição ser referência para tratamento cirúrgico de pacientes com valvopatia e aortopatia, podendo assim não representar fielmente o comportamento da doença na população geral, mas nos faz entender melhor as características da patologia em uma população altamente complexa. Além disso, o curto período de inclusão (2014 a 2019) assegurou a homogeneidade de técnicas cirúrgicas e de recomendações de intervenção. Outro ponto a ser mencionado é que não foi realizada, de maneira rotineira, a análise histopatológica da aorta nos pacientes do nosso estudo. Entretanto, devido à alta associação de aortopatia com $\mathrm{VAB}$, demonstrada em estudos prévios, podemos inferir que as alterações da aorta são relacionadas à valvopatia. ${ }^{6,7,14}$

\section{Conclusão}

Em pacientes com VAB abordados no nosso serviço foi identificada uma maior incidência de aortopatia que o descrito na literatura, evidenciando a heterogeneidade sindrômica da $\mathrm{VAB}$ e a necessidade adicional de avaliação da aorta com tomografia computadorizada ou ressonância magnética em portadores de VAB.

\section{Contribuição dos autores}

Concepção e desenho da pesquisa, Análise e interpretação dos dados: Kirschbaum M, Rosa VEE, Fernandes JRC, Santis A,

\section{Referências}

1. Osler W. The bicuspid condition of the aortic valve. Trans Assoc Am Physicians. 1886;2:185-92.

2. Roberts WC. The Congenitally Bicuspid Aortic Valve. Astudy of 85 autopsy cases. Am J Cardiol. 1970;26(1):72-83. doi: 10.1016/0002-9149(70)90761-7.

3. Larson EW, Edwards WD. Risk Factors for Aortic Dissection: A Necropsy Study of 161 Cases. Am J Cardiol. 1984;53(6):849-55. doi: 10.1016/00029149(84)90418-1.

4. Ozturk C, Ozturk A, Balta S, Aparci M, Demirkol S, Unlu M, et al. Direction of Aortic Jet Flow is Important in Predicting Aortic Dilatation in Patients with Bicuspid Aortic Valve. Kardiol Pol. 2016;74(2):196. doi: 10.5603/ KP.2016.0025

5. Shin HJ, Shin JK, Chee HK, Kim JS, Ko SM. Characteristics of Aortic Valve Dysfunction and Ascending Aorta Dimensions According to Bicuspid Aortic Valve Morphology. Eur Radiol. 2015;25(7):2103-14. doi: 10.1007/s00330014-3585-z.

6. Fedak PW, Sa MP, Verma S, Nili N, Kazemian P, Butany J, et al. Vascular Matrix Remodeling in Patients with Bicuspid Aortic Valve Malformations: Implications for Aortic Dilatation. J Thorac Cardiovasc Surg. 2003;126(3):797-806. doi: 10.1016/s0022-5223(03)00398-2.

7. Fedak PW, Verma S, David TE, Leask RL, Weisel RD, Butany J. Clinical and Pathophysiological Implications of a Bicuspid Aortic Valve. Circulation. 2002;106(8):900-4. doi: 10.1161/01.cir.0000027905.26586.e8.

8. Tzemos N, Therrien J, Yip J, Thanassoulis G, Tremblay S, Jamorski MT, et al Outcomes in Adults with Bicuspid Aortic Valves. JAMA. 2008;300(11):131725. doi: 10.1001/jama.300.11.1317.
Accorsi TD, Sampaio RO, Tarasoutchi F; Obtenção de dados: Kirschbaum M, Sampaio BPA, Thevenard G, Quintanilha NR; Análise estatística e Redação do manuscrito: Kirschbaum M, Rosa VEE, Tarasoutchi F; Revisão crítica do manuscrito quanto ao conteúdo intelectual importante: Kirschbaum M, Rosa VEE, Sampaio BPA, Thevenard G, Quintanilha NR, Fernandes JRC, Santis A, Accorsi TD, Sampaio RO, Tarasoutchi F.

\section{Potencial conflito de interesse}

Não há conflito com o presente artigo

\section{Fontes de financiamento}

O presente estudo não teve fontes de financiamento externas.

\section{Vinculação acadêmica}

Não há vinculação deste estudo a programas de pósgraduação.

\section{Aprovação ética e consentimento informado}

Este estudo foi aprovado pelo Comitê de Ética do USP - Hospital das Clínicas da Faculdade de Medicina da Universidade de São Paulo - HCFMUSP sob o número de protocolo SDC 5094/20/123. Todos os procedimentos envolvidos nesse estudo estão de acordo com a Declaração de Helsinki de 1975, atualizada em 2013.

9. Tarasoutchi F, Montera MW, Ramos AIO, Sampaio RO, Rosa VEE, Accorsi TAD, et al. Atualização das Diretrizes Brasileiras de Valvopatias: Abordagem das Lesões Anatomicamente Importantes. Arq Bras Cardiol. 2017;109(6 suppl 2):1-34. doi: 10.5935/abc.20180007.

10. Erbel R, Aboyans V, Boileau C, Bossone E, Bartolomeo RD, Eggebrecht H, et al. 2014 ESC Guidelines on the Diagnosis and Treatment of Aortic Diseases: Document Covering Acute and Chronic Aortic Diseases of the Thoracic and Abdominal Aorta of the Adult. The Task Force for the Diagnosis and Treatment of Aortic Diseases of the European Society of Cardiology (ESC). Eur Heart J. 2014;35(41):2873-926. doi: 10.1093/eurheartj/ehu281.

11. Fernández B, Fernández MC, Durán AC, López D, Martire A, Sans-Coma V. Anatomy and Formation of Congenital Bicuspid and Quadricuspid Pulmonary Valves in Syrian Hamsters. Anat Rec. 1998;250(1):70-9. doi: 10.1002/(SICI)1097-0185(199801)250:1<70::AID-AR7>3.0.CO;2-I.

12. Kappetein AP, Groot ACG, Zwinderman AH, Rohmer J, Poelmann RE, Huysmans HA. The Neural Crest as a Possible Pathogenetic Factor in Coarctation of the Aorta and Bicuspid Aortic Valve. J Thorac Cardiovasc Surg. 1991;102(6):830-6

13. Sans-Coma V, Fernández B, Durán AC, Thiene G, ArquéJM, Muñoz-Chápul $\mathrm{R}$, et al. Fusion of Valve Cushions as a Key Factor in the Formation of Congenital Bicuspid Aortic Valves in Syrian Hamsters. Anat Rec. 1996;244(4):490-8. doi: 10.1002/(SICI)1097-0185(199604)244:4<490::AID-AR7>3.0.CO;2-Z.

14. Andrei AC, Yadlapati A, Malaisrie SC, Puthumana JJ, Li Z, Rigolin VH, et al Comparison of Outcomes and Presentation in Men-versus-Women with Bicuspid Aortic Valves Undergoing Aortic Valve Replacement. Am J Cardiol. 2015;116(2):250-5. doi: 10.1016/j.amjcard.2015.04.017. 
15. Hiratzka LF, Bakris GL, Beckman JA, Bersin RM, Carr VF, Casey DE, et al. 2010 ACCF/AHA/AATS/ACR/ASA/SCA/SCAI/SIR/STS/SVM Guidelines for the Diagnosis and Management of Patients with Thoracic Aortic Disease. A Report of the American College of Cardiology Foundation/American Heart Association Task Force on Practice Guidelines, American Association for Thoracic Surgery, American College of Radiology,American Stroke Association, Society of Cardiovascular Anesthesiologists, Society for Cardiovascular Angiography and Interventions, Society of Interventional Radiology, Society of Thoracic Surgeons, and Society for Vascular Medicine. Circulation. 2010;121(13):266-369. doi: 10.1016/j.jacc.2010.02.015

16. Vendramin I, Meneguzzi M, Sponga S, Deroma L, Cimarosti R, Lutman C, et al. Bicuspid Aortic Valve Disease and Ascending Aortic Aneurysm: Should an Aortic Root Replacement be Mandatory?. Eur J Cardiothorac Surg. 2016;49(1):103-9. doi: 10.1093/ejcts/ezv069.

17. Girdauskas E, Disha K, Borger MA, Kuntze T. Long-term Prognosis of Ascending Aortic Aneurysm After Aortic Valve Replacement for Bicuspid Versus Tricuspid Aortic Valve Stenosis. J Thorac Cardiovasc Surg. 2014;147(1):276-82. doi: 10.1016/j.jtcvs.2012.11.004.
18. Lee SH, Kim JB, Kim DH, Jung SH, Choo SJ, Chung CH, et al. Management of Dilated Ascending Aorta During Aortic Valve Replacement: Valve Replacement Alone Versus Aorta Wrapping Versus Aorta Replacement. J Thorac Cardiovasc Surg. 2013;146(4):802-9. doi: 10.1016/j. jtcvs.2013.06.007.

19. Michelena HI, Desjardins VA, Avierinos JF, Russo A, Nkomo VT, Sundt TM, et al. Natural History of Asymptomatic Patients with Normally Functioning or Minimally Dysfunctional Bicuspid Aortic Valve in the Community. Circulation. 2008;117(21):2776-84. doi: 10.1161/ CIRCULATIONAHA.107.740878.

20. Michelena HI, Khanna AD, Mahoney D, Margaryan E, Topilsky Y, Suri RM, et al. Incidence of Aortic Complications in Patients with Bicuspid Aortic Valves. JAMA. 2011;306(10):1104-12. doi: 10.1001/jama.2011.1286.

21. Masri A, Kalahasti V, Alkharabsheh S, Svensson LG, Sabik JF, Roselli EE, et al. Characteristics and Long-term Outcomes of Contemporary Patients with Bicuspid Aortic Valves. J Thorac Cardiovasc Surg. 2016;151(6):1650-9. doi: 10.1016/j.jtcvs.2015.12.019.

\section{* Material suplementar}

Para informação adicional, por favor, clique aqui. 